\title{
Mini-Review: Factors Affecting Multiple Invasions of Erythrocytes in Plasmodium and other Malaria-like Parasites. A Neglected Characteristic of Infections
}

\author{
Josué Martínez-de la Puente ${ }^{1, *}$ and Santiago Merino ${ }^{1}$
}

\begin{abstract}
Departamento de Ecología Evolutiva, Museo Nacional de Ciencias Naturales - C.S.I.C., C José Gutiérrez Abascal 2, E-28006 Madrid, Spain
\end{abstract}

\begin{abstract}
Multiple invasions (MIs) are produced when the same erythrocyte is infected by more than one parasite cell. These MIs commonly occur in different haemosporidia species including Plasmodium and other malaria-like parasites. However, the frequency of MIs has been traditionally considered in studies both in vivo and in vitro as an artefact produced by high parasite densities, leading most researchers to think that MIs does not have a true biological meaning but they are merely the product of chance. Other proposed explanations for the occurrence of MIs include an adaptive host strategy to reduce parasite damage and hinder parasite transmission success and an adaptive parasite strategy which favours parasite transmission success. Here we review the relevant literature supporting or rejecting these hypotheses proposed to explain the occurrence of MIs. Although the possibility that MIs being due to higher parasite densities has received much support, more studies are clearly necessary to reveal the potential importance of host defences and parasite strategies on the occurrence of MIs in nature.
\end{abstract}

Keywords: Haemoproteus, Leucocytozoon, Malaria, Host-parasite interactions, Transmission.

\section{INTRODUCTION}

Haemospororins, or malaria-like parasites, infect bloodsucking dipterans and several classes of vertebrates. The life cycle of these parasites includes both sexual and asexual phases. Within the vertebrate hosts, after undergoing asexual reproduction, each merozoite invades a red blood cell (RBC), developing into either a gametocyte (gamete precursors) or a meront (schizonts). In some species, such as Plasmodium, both meronts and gametocytes can be found infecting RBCs, and in other species, such as Haemoproteus and Leucocytozoon, meronts do not infect erythrocytes.

In the vertebrate host, each infected RBC usually contains only one parasite cell but, however, multiply invaded RBCs are also found. Many studies have found MIs in nature with cases reported for all Plasmodium species infecting humans [1, 2, 3] and even for several Plasmodium species infecting non-human hosts [4,5]. MIs are also produced by other malaria-like haemospororins including Haemoproteus $[6,7,8]$ and Leucocytozoon species [5] infecting birds and reptiles. Also, reports of MIs by other blood parasites with intraerythrocytic stages, such as Hepatozoon [9,10,11], Babesia [12, 13] and Karyolysus [14] exist in parasitological literature.

Traditionally, the frequency of multiple invasions (MIs) has been considered both in studies in vivo and in vitro as an artefact produced by high parasite densities, with the intensity (density) of infection as the main factor determining their occurrence $[1,15]$. In this respect, the Poisson distribution has been proposed as an adequate model to predict the

*Address correspondence to this author at Departamento de Ecología Evolutiva, Museo Nacional de Ciencias Naturales - C.S.I.C., Cl José Gutiérrez Abascal 2, E-28006 Madrid, Spain; Tel: +34914111328; Fax: +34 915645 078; E-mail: jmp@mncn.csic.es abundance of MIs according to the density of gametocytes [16]. Also, differences in the intensity of infection could be the key factor explaining differences in the occurrence of MIs between Plasmodium species infecting humans. For example, MIs are more commonly observed in $P$. falciparum infections [1] while $P$. vivax MIs have been considered as an uncommon phenomenon (see references in [17]). Therefore the higher densities of infection that usually occur in patients infected by $P$. falciparum as compared to patients infected by $P$. vivax may explain, at least in part, these differences. In fact, when $P$. vivax reaches high parasitaemias (high intensities of infection), MIs are also found [18, 19]. Thus, the role of the parasite density (intensity of infection) as the main factor affecting the occurrence of MIs has received considerable support. However other studies suggest that parasites and host related factors could be also important to determine the occurrence of MIs in nature (Fig. 1).

Recently, Jovani [20] proposed that MIs, even when produced at random, could be a way to increase parasite transmission because infections of male-female gametocytes into the same RBC could ensure an encounter between gametes into the gut of mosquito vector. In this sense, co-invasion of male and female gametocytes could be a parasite adaptive strategy to increase their transmission success [15]. However, if multiple invasions are an adaptive strategy by the parasite to facilitate gametocyte encounter, MIs should fulfil several predictions [15] according to fertility insurance hypothesis [21]: i) male and female gametocytes in MIs must reach maturity to be capable to undergo sexual reproduction in the insect vector, ii) MIs must be produced preferentially by gametocytes of different sexes and iii) the frequency of MIs must increase as parasitaemia (intensity of infection) in the vertebrate host decrease, because the higher difficulty of male and female gametocyte to meet in the insect vector increase as the number of gametes being ingested in the vector 


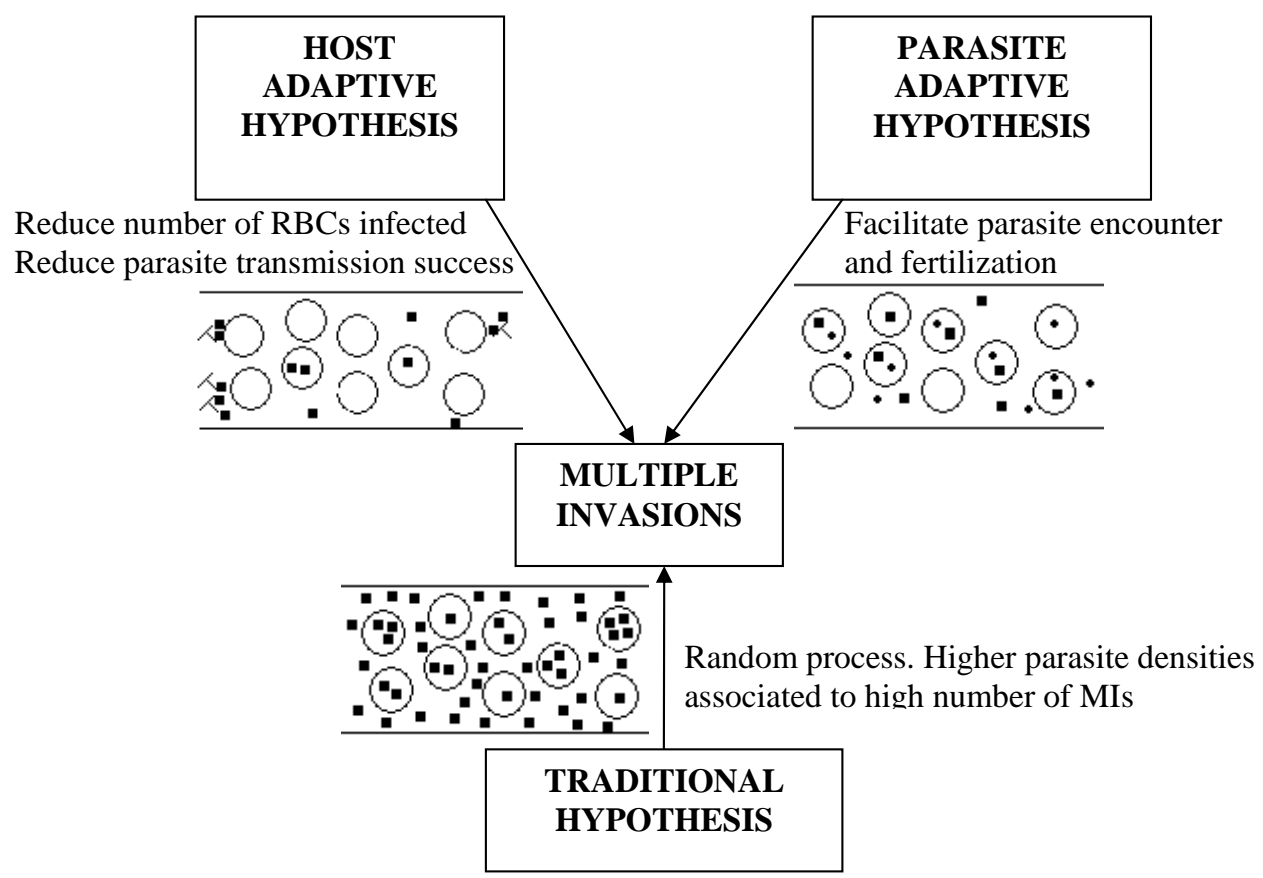

Fig. (1). Hypotheses proposed to explain the occurrence of MIs. According to the host adaptive hypothesis, host antibodies could cross-link parasites and promote MIs to reduce the number of single invasions and increase the difficulties of parasite to reach maturity at the same time that may reduce the parasite probability of transmission (parasites are represented as small squares and RBCs as empty circles). The parasite adaptive hypothesis implies that parasites promote the occurrence of MIs comprising gametocytes of different sexes (represented as small squares and points) to facilitate parasite encounter and fertilization in the insect vector. Alternatively, according to the traditional hypothesis, the occurrence of MIs could be a random process exclusively dependent of the parasite density of infection, with higher number of MIs as parasitaemia increase.

blood meal decrease. A recent study using an avian malarialike model did not support these predictions [15], perhaps because Haemoproteus parasites are able to develop other strategies to assure fertilization in the vector [22]. However, infection by $P$. falciparum parasites from patients who displayed uncomplicated malaria and supported parasitaemias lower than $10 \%$ of the total RBC, produced a higher proportion of MIs than did those infections that caused severe or cerebral malaria [23, 24] where density of parasites is usually high. This result may support that MIs could be selected for in parasite species or isolates where gametocyte density is always low enough to compromise parasite fertilization success. If those parasites that caused uncomplicated malaria produced mature male-female gametocyte infections, they could maintain transmission and sexual reproduction success at lower parasite densities. In addition, these parasites could increase the lifespan of their host, by reduced cell invasion and destruction, and consequently increase the time available for successful parasite transmission.

On the other hand, different studies have found evidences for the role of immunological defences affecting the occurrence of MIs [25, 26, 27]. Hosts mediated factors promoting MIs may imply beneficial effects for them due to the reduction of the total number of RBCs infected inside the individual host. In addition, due to the difficulties of parasites to reach maturity in MIs [15, 28], the host induction of MIs could render an indirect benefit for hosts by reducing the parasite transmission efficacy and therefore the probability of infection or reinfections in the population of hosts and reservoirs. This possibility could evolve not for a group se- lection process but for the potential selection of lower virulent parasite strains in hosts when the transmission efficacy of the parasite is reduced [29]. That is, if transmission efficacy of the parasite is lowered by host responses, a less virulent, more chronic disease with longer infection (and transmission) time will be favoured. Alternatively, the positive association between antibodies and MIs could reflect the fact that parasites in MIs survive poorly and as a result, they stimulate the development of the host immune system more than single-infecting parasites. The first evidence for these possibilities was provided by Miller et al. [25] who studied Plasmodium knowlesi, and reported an increase in the occurrence of MIs associated with the presence of the monoclonal antibody $(\mathrm{mAb})$ against to a $P$. knowlesi protein. Subsequent studies, both in laboratory and nature, also support the role of host immune system promoting multiple invasions [26, 27] but see [30]. Two mechanisms for MIs being produced by immune response have been suggested: (i) as the result of the invasion together of parasites cross-linked by the same antibody; or (ii) by several antibody-dissociated merozoites in close proximity to the same RBC [31]. More experimental studies in vivo are necessary to reveal the actual effect of antibodies on MIs in nature. In addition, other hosts related factors such as host age (M. A. Peirce pers. comm.) or sex could also play a role in the occurrence of MIs by the association of those variables with both, the parasite intensity of infection and the immune response of hosts. Other factors such as differential susceptibility of RBCs to infection or erythrocyte abnormalities such as thalassemia could also affect the occurrence of MIs. 
MIs are a characteristic of infections by haemosporidian parasites in blood cells from vertebrate hosts with potential important consequences for the success of the parasite and/or the host. Although many reports exist for the occurrence of MIs in nature, the majority of them could be considered as case reports and more studies on the role of host immune system or the molecular selectivity affecting the occurrence of MIs should be done in order to clarify their role in these infections. In this respect, it is especially interesting to understand the host-related mechanisms involved in the occurrence of MIs potencially reducing the parasite transmission success and the negative effects of parasites on hosts. To test some of these possibilities, researchers could use samples obtained in other previous studies such as blood smears from human malaria patients. These smears could be employed to study in depth the random or non-random distribution of MIs, because usually blood samples are available before and after antimalaria medication treatments. In those cases, under the parasite adaptive hypothesis, we could expect that the number of MIs should increase as parasite density is reduced by medication.

\section{ACKNOWLEDGEMENTS}

The study was financially supported by project CGL 2006-14129-C02-01 from the Spanish Ministerio de Educación y Ciencia. J. Martínez-de la Puente is supported by a "El Ventorrillo" grant. Authors thank M. A. Peirce for his comments about multiple invasions. Comments by two anonymous reviewers greatly improved a earlier version of the manuscript.

\section{REFERENCES}

[1] Wang CC. Multiple invasion of erythrocyte by malaria parasites. Trans R Soc Trop Med Hyg 1970; 64: 268-270.

[2] Poirriez J, Landau I, Verhaeghe A, Snounou G, Dei-Cas E. Les formes atypiques de Plasmodium vivax a propos d'une observation. Ann Parasitol Hum Comp 1991; 66: 149-154.

[3] Marzars E, Laberenne JE, Snounou G, Dei-Cas E, Cattoen C, Poirriez, J. Relapsing Plasmodium vivax malaria with atypical parasite forms and phagocytosis by peripheral neutrophils. Parasite 1997; 4: 263-267.

[4] Bungener W. Further observations of the course of Plasmodium berghei infection in the mouse (in German). Tropenmed Parasitol 1979; 30: 24-34.

[5] Valkiūnas G. Avian malaria parasites and other haemosporidia. Florida: CRC Press 2005.

[6] Ahmed FE, Mohammed AHH. Studies of growth and development of gametocytes in Haemoproteus columbae Kruse. J Protozool 1978; 25: 174-177.

[7] Lainson R, Naiff RD. Haemoproteus (Apicomplexa : Haemoproteidae) of tortoises and turtles. Proc R Soc Lond Ser B 1998; 265 : 941-949.

[8] Jovani R, Amo L, Arriero E, Krone O, Marzal A, Shurunlinkov P, Tomás G, Sol D, Hagen J, López P, Martín J, Navarro C, Torres J. Double gametocyte infections in apicomplexan parasites of birds and reptiles. Parasitol Res 2004; 94: 155-157.

[9] Mackerras MJ. The haematozoa of australian mammals. Aust J Zool 1959; 7: 105-135.

[10] Bettiol SS, Goldsmid JM, Le DD, Driessen M. The first record of a member of the genus Hepatozoon in the Eastern Barred Bandicoot (Perameles gunnii) in Tasmania. J Parasitol 1996; 82: 829-830.
[11] Lainson R, Paperna L, Naiff RD. Development of Hepatozoon caimani (Carini, 1909) Pessôa, De Biasi \& De Souza, 1972 in the caiman Caiman c. crocodiles, the frog Rana catesbeiana and the mosquito Culex fatigans. Mem Inst Oswaldo Cruz 2003; 98: 103113.

[12] Merino S. Babesia bennetti sp. nov., from the yellow-legged gull (Larus cachinnans, Aves, Laridae) in Benidorm Island, Mediterranean sea. J Parasitol 1998; 84: 422-424.

[13] Peirce MA. A taxonomic review of avian piroplasms of the genus Babesia Starcovici, 1893 (Apicomplexa: Piroplasmorida: Babesiidae). J Nat Hist 2000; 34: 317-332.

[14] Hussein A-NA. Light and transmission electron microscopic studies of a haemogregarine in naturally infected fan-footed gecko (Ptyodactylus hasselquistii). Parasitol Res 2006; 98: 468-471.

[15] Martínez-de la Puente J, Merino S, Tomás G, Moreno J, Morales J, Lobato E. Are multiple gametocyte infections in malarial parasites an adaptation to ensure fertility? Parasitology 2006; 132: 23-28.

[16] Jovani R, Sol D. How predictable is the abundance of double gametocyte infections? Parasitol Res 2005; 97: 84-86.

[17] Prasad RN, Prasad H, Virk KJ, Sharma VP. Detection of multiple invasión of erythrocytes by Plasmodium vivax. Trans R Soc Trop Med Hyg 1990; 41: 437-438.

[18] Witzig RS, Barker R H jr. Atypical Plasmodium vivax infection in Peru. Trans R Soc Trop Med Hyg 1994; 88: 198.

[19] Poirriez J, Snounou G, Blanckaert D. Multiple invasion of red blood cells by Plasmodium vivax in vivo. Trans R Soc Trop Med Hyg 1995; 89: 509-510.

[20] Jovani R. Malaria transmission, sex ratio and erythrocytes with two gametocytes. Trends Parasitol 2002; 18: 537-539.

[21] West SA, Smith TG, Nee S, Read AF. Fertility insurance and the sex ratios of malaria and related hemospororin blood parasites. J Parasitol 2002; 88: 258-263.

[22] Merino S, Tomás G, Moreno J, Sanz J J, Arriero E, Folgueira C. Changes in Haemoproteus sex ratios: fertility insurance or differential sex life-span? Proc R Soc Lond B 2004; 271: 1605-1609.

[23] Simpson JA, Silamut K, Chotivanich K, Pukrittayakamee S, White NJ. Red cell selectivity in malaria: study of multiple-infected erythrocytes. Trans R Soc Trop Med Hyg 1999; 93: 165-168.

[24] Chotivanich K, Udomsangpetch R, Simpson JA, Newton P, Pukrittayakamee S, Looareesuwan S, White NJ. Parasite multiplication potential and the severity of falciparum malaria. J Infect Dis 2000; 181: 1206-1209.

[25] Miller LH, David PH, Hudson DE, Hadley TJ, Richards RL, Aikawa M. Monoclonal antibodies to a $140,000 \mathrm{~m}$.W. protein on Plasmodium knowlesi merozoites inhibit their invasion of rhesus erythrocytes. J Immunol 1984; 132: 438-442.

[26] Ramasamy R, Yasawardena S, Kanagaratnam R, Buratti E, Baralle FE. Antibodies to a merozoite surface protein promote multiple invasion of red blood cells by malaria parasites. Parasite Immunol 1999; 21: 397-407.

[27] Martínez-de la Puente J, Merino S, Tomás G, Moreno J, Morales J, Lobato E, Martínez, J, García-Fraile S. Can the host immune system promote multiple invasions of erythrocytes in vivo? Differential effects of medication treatment and host sex in a wild malarialike model. Parasitology 2007; 134: 651-655.

[28] Inselburg J. Gametocyte formation by the progeny of single Plasmodium falciparum schizonts. J Parasitol 1983; 69: 584-591.

[29] Ewald, P.W. Evolution of Infectious Diseases. Oxford: Oxford University Press 1994.

[30] Frazén L, Wahlin B, Wahlgren M, Aslund L, Perlmann P, Wigzell, $\mathrm{H}$, Pettersson U. Enhancement or inhibition of Plasmodium falciparum erythrocyte reinvasion in vitro antibodies to an asparagine rich protein. Mol Biochem Parasitol 1989; 32: 201-212.

[31] Ramasamy R, Ramasamy M, Yasawardena S. Antibodies and Plasmodium falciparum merozoites. Trends Parasitol 2001; 17: 194-197. 